\title{
On the numerical solution of Hammerstein integral equations using shifted Chebyshev polynomials of the third kind method
}

\author{
Amr M. S. Mahdy ${ }^{1}$ and Emad M.H.Mohamed ${ }^{2}$ \\ ${ }^{1}$ Mathematics Department, Faculty of Science, Zagazig University, Zagazig City, Egypt \\ ${ }^{2}$ Department of Mathematics, Faculty of Science, Alazhar University, Cairo, Egypt
}

Received: 18 January 2017, Accepted: 27 February 2017

Published online: 26 August 2017.

\begin{abstract}
In this paper, shifted Chebyshev polynomials of the third kind method is presented to solve numerically the Fredholm, Volterra-Hammerstein integral equations. The proposed method converts the equation system of linear or non-linear algebraic equations, which can be solved. Some numerical examples are included to demonstrate the validity and applicability of the proposed technique. All computations are done using Mathematica 7.
\end{abstract}

Keywords: Fredholm-Hammerstein integral equations, Volterra integral equation, shifted Chebyshev polynomials of the third kind method.

\section{Introduction}

Integral equations have proved itself as one of the most important branches of mathematics. Integral equations are one of the most useful mathematical tools in both pure and applied mathematics. The theory of integral equations has close contacts with many different areas of mathematics. Foremost among these are differential equations and operator theory. Many problems in the fields of ordinary and partial differential equations can be recast as integral equations ([1], [3], [4], [18]). Integral equations arise naturally in physics, chemistry, biology, engineering and many physical phenomena ([1], [5]). The principle investigators of the theory of integral equations are Vito Volterra (1860-1940) and Ivar Fredholm (1866-1927), together with David Hilbert (1862-1943) and Erhand Schmidt (1876-1959). There are several methods for approximating the solution of linear and non-linear integral equations ([10]-[14]).

In [6] the author using Legendre pseudo-spectral method to approximate and exact solutions of the fractional-order delay differential equations. In [7] the author using Legendre pseudo-spectral method for solving the fractional diffusion equation. In [9] the author using implementation of the operational matrix of fractional derivative for solving nonlinear multi-order fractional differential equations. In [16] the author using homotopy analysis method for solving the bi-harmonic equation.

The Chebyshev polynomials are mostly used to solve problems of differential equations or integral equations. Chebyshev polynomials are used to introduce an efficient medication of homotopy perturbation method [8]. Also, the polynomial approximation is used to solve high-order linear Fredholm integro-differential equations with constant coefficient [11] and others ([15], [17]). 
We consider the Hammerstein integral equations in the forms [17]

$$
x(t)=f(t)+\lambda_{1} \int_{0}^{1} k_{1}(t, s) F\left(x(s) d s+\lambda_{2} \int_{0}^{t} k_{2}(t, s) G(x(s)) d s\right.
$$

where $f(t), k_{1}(t, s)$ and $k_{2}(t, s)$ are given functions, $t \leq 0, s \leq 1$ and $\lambda_{1}, \lambda_{2}$ are arbitrary constant.

\section{Some Properties of Chebyshev Polynomials of the Third Kind}

\subsection{Chebyshev Polynomials of the Third Kind}

The Chebyshev polynomials $V_{n}(x)$ of the third kind are orthogonal polynomials of degree $n$ in $x$ defined on $[-1,1][2,20]$.

$$
V_{n}=\frac{\cos (n+1 / 2) \Theta}{\cos \frac{\Theta}{2}}
$$

where $x=\cos \Theta$ and $\Theta \in[0, \pi]$. The polynomials $V_{n}(x)$ are orthogonal on $[-1,1]$ with respect to the inner product:

$$
\left\langle V_{n}(x), V_{m}(x)\right\rangle=\int_{-1}^{1} \sqrt{\frac{1+x}{1-x}} V_{n}(x) V_{m}(x) d x=\left\{\begin{array}{l}
\pi, \text { for } n=m \\
0, \text { for } n \neq m
\end{array}\right.
$$

where $\sqrt{\frac{1+x}{1-x}}$ is weight function corresponding to $V_{n}(x)$. The polynomials $V_{n}(x)$ may be generated by using the recurrence relations

$$
\begin{aligned}
& V_{n+1}(x)=2 x V_{n}(x)-V_{n-1}(x), \\
& V_{0}(x)=1, V_{1}(x)=2 x-1, n=1,2, \cdots
\end{aligned}
$$

The analytical form of the Chebyshev polynomials of the third kind $V_{n}(x)$ of degree $n$ are given by

$$
V_{n}(x)=\sum_{k=0}^{\left[\frac{2 n+1}{2}\right]}(-1)^{k}(2)^{n-k} \frac{(2 n+1) \Gamma(2 n-k+1)}{\Gamma(k+1) \Gamma(2 n-2 k+2)}(x+1)^{n-k}, n \in Z^{+}
$$

where $\left[\frac{2 n+1}{2}\right]$ denotes the integer part of $\frac{(2 n+1)}{2}$.

\subsection{The shifted Chebyshev polynomials of the third kind}

In order to use the these polynomials on the interval $[0,1]$, we define the so called shifted Chebyshev polynomials of the third kind by the introducing the change of variable $V(x)=2 x-1$ [20]. The shifted Chebyshev polynomials of the third kind are define as $V_{n}^{*}(x)=V_{n}(2 x-1)$.

These polynomials are orthogonal on the support interval $[0,1]$ as the following inner product

$$
\left\langle V_{n}^{*}(x), V_{m}^{*}(x)\right\rangle=\int_{-1}^{1} \sqrt{\frac{1+x}{1-x}} V_{n}^{*}(x) V_{m}^{*}(x) d x=\left\{\begin{array}{l}
\frac{\pi}{2}, \text { for } n=m \\
0, \text { for } n \neq m
\end{array}\right.
$$


where $\sqrt{\frac{x}{1-x}}$ is the weight function corresponding to $V_{n}^{*}(x)$ and normalized by the requirement that $V_{n}^{*}(1)=1$.

The polynomials $V_{n}^{*}(x)$ may be generated by using the recurrence relations

$$
\begin{aligned}
& V_{n-1}^{*}(x)=2(2 x-1) V_{n}^{*}(x)-V_{n-1}^{*}(x), \\
& V_{0}^{*}(x)=1, V_{1}^{*}(x)=4 x-3, n=1,2, \cdots
\end{aligned}
$$

The analytical form of the shifted Chebyshev polynomials of the third kind $V_{n}^{*}(x)$ of degree $n$ in $x$ given by:

$$
V_{n}^{*}(x)=\sum_{k=0}^{n}(-1)^{k}(2)^{2 n-2 k} \frac{(2 n+1) \Gamma(2 n-k+1)}{\Gamma(k+1) \Gamma(2 n-2 k+2)}(x)^{n-k}, n \in Z^{+},
$$

In the special method, the square integrable function $g(x)$ in $[0,1]$, is represented by an infinite expansion of the shifted Chebyshev polynomials of the third kind as following:

$$
g(x)=\sum_{i=0}^{\infty} b i V_{i}^{*}(x)
$$

where $b_{i}$ is a chosen sequence of prescribed basis function. One then proceeds some how to estimate as many as possible of the coefficients $b_{i}$, thus approximating $g(x)$ by a finite sum of $(m+1)$-terms such as:

$$
g_{m}(x)=\sum_{i=0}^{m} b i V_{i}^{*}(x)
$$

where the coefficients $b_{i}, i=0,1, \cdots$ are given by

$$
b_{i}=\frac{1}{\pi} \int_{-1}^{1} g\left(\frac{x+1}{2} V_{i}(x) \sqrt{\frac{1+x}{1-x}} d x,\right)
$$

where the coefficients $b_{i}, i=0,1, \cdots$ are given by

$$
b_{i}=\frac{2}{\pi} \int_{0}^{1} g(x) V_{i}^{*}(x) \sqrt{\frac{x}{1-x}} d x,
$$

\section{Procedure Solution Using the Proposed Numerical Method}

We consider the Fredholm-Volterra integral equation (1). The function $x(t)$ may be expanded by infinite series of the shifted Chebyshev polynomials of the third kind as follows [17]:

$$
x(t)=\sum_{n=0}^{\infty} c_{n} V_{n}^{*}(t),
$$

where $c_{n}=\left(x(t), V_{n}^{*}(t)\right)$. If we consider truncation series in Eq.(10), we obtain

$$
\begin{aligned}
x(t) & \simeq \sum_{n=0}^{N} c_{n} V_{n}^{*}(t) \\
& =c^{T} V^{*}(t),
\end{aligned}
$$


such that $c$ and $V^{*}(t)$ are matrices given by

$$
c=\left[c_{0}, c_{1}, \cdots, c_{N}\right], \quad V^{*}(t)=\left[V_{0}^{*}(t), V_{1}^{*}(t), \cdots, V_{N}^{*}(t)\right]^{T} .
$$

Then, we substitute the approximation Eq.(11) into Eq.(1), we get

$$
\sum_{n=0}^{N} c_{n} V_{n}^{*}(t)=f(t)+\lambda_{1} \int_{0}^{1} k_{1}(t, s) F\left(c^{T} V^{*}(s)\right) d s+\lambda_{2} \int_{0}^{t} k_{2}(t, s) G\left(c^{T} V^{*}(s)\right) d s .
$$

Now, to use the shifted Chebyshev polynomials of the third kind which is a matrix method based on the shifted Chebyshev polynomials points depended by

$$
t_{i}=-1+\frac{2}{N} i, \quad i=0,1,2, \cdots, N .
$$

We collocate Eq.(13) with the points Eq.(14) to obtain

$$
\sum_{n=0}^{N} c_{n} V_{n}^{*}\left(t_{j}\right)=f\left(t_{j}\right)+\lambda_{1} \int_{0}^{1} k_{1}\left(t_{j}, s\right) F\left(c^{T} V^{*}(s)\right) d s+\lambda_{2} \int_{0}^{t_{j}} k_{2}\left(t_{j}, s\right) G\left(c^{T} V^{*}(s)\right) d s .
$$

The integral term in Eq.(15) can be found using composite trapezoidal integration technique as.

$$
\int_{0}^{1} k_{1}\left(t_{j}, s\right) F\left(c^{T} V^{*}(s)\right) d s \cong \frac{h}{2}\left[\Omega_{1}\left(S_{0}\right)+\Omega_{1}\left(S_{m}\right)+2 \sum_{k-1}^{m-1} \Omega_{1}\left(S_{k}\right)\right],
$$

where $\Omega_{1}(S)=k_{1}\left(t_{j}, s\right) F\left(c^{T} V^{*}(s)\right), h=\frac{1}{m}$, for an arbitrary integer $m, S_{i}=i h, i=0,1, \cdots, m$ and

$$
\int_{0}^{t_{j}} k_{2}\left(t_{j}, s\right) G\left(c^{T} V^{*}(s)\right) d s \cong \frac{h_{j}}{2}\left[\Omega_{2}\left(\bar{S}_{0}\right)+\Omega_{2}\left(\bar{S}_{m}\right)+2 \sum_{k-1}^{m-1} \Omega_{2}\left(\bar{S}_{k}\right)\right],
$$

where $\Omega_{2}(S)=k_{2}\left(t_{j}, s\right) G\left(c^{T} V^{*}(s)\right), h_{j}=\frac{t_{j}}{m}$, for an arbitrary integer $m, \bar{S}_{i}=i h, i=0,1, \cdots, m$. Eq.(15) gives $(N+1)$ system of linear or non-linear algebraic equations, which can be solved for $c_{k}, k=0,1, \cdots, N$. So the unknown function $x(t)$ can be found.

\section{Numerical Implementation}

In this section to a chive the validity, the accuracy and our theoretical discussion of the proposed method we give some computational results of numerical examples.

Example 1. Consider Eq.(1) with the following functions and coefficients [17]

$$
\begin{gathered}
f(t)=t^{3}-(6-2 e) e^{t}, \quad \lambda_{1}=1, \quad \lambda_{2}=0 \\
k_{1}(t, s)=e^{t+s}, \quad k_{2}(t, s)=0, \quad F(x(s))=x(s), \quad G(x(s))=0
\end{gathered}
$$

Eq.(1) takes the form

$$
x(t)=t^{3}-(6-2 e) e^{t}+\int_{0}^{1} e^{(s+t)} x(s) d s
$$


We apply the suggested method with $N=4$ and approximate the solution $x(t)$ as follows

$$
x_{N}(t)=\sum_{i=0}^{4} c_{i} V_{i}^{*}(t) .
$$

By the same procedure in the previous section and using Eq.(15) we have

$$
\sum_{i=0}^{4} c_{i} V_{i}^{*}\left(t_{j}\right)-\frac{h}{2}\left[\Omega\left(s_{0}\right)+\Omega\left(s_{m}\right)+2 \sum_{k=1}^{m-1} \Omega\left(s_{k}\right)\right]=f\left(t_{j}\right), \quad j=0,1,2,3,4
$$

where

$$
\Omega(s)=e^{\left(s+t_{j}\right)} \sum_{i=0}^{4} c_{i} V_{i}^{*}(s)
$$

and the nodes $s_{i+1}=s_{i}+h, i=0,1, \cdots, m, s_{0}=0$ and $h=\frac{1}{m}$. Eq.(20) represents linear system of $N+1$ algebraic equations in the coefficients $c_{i}$.

By solving it using the conjugate gradient method, we obtain

$$
c_{0}=0.70894, c_{1}=0.251995, c_{2}=0.03710, c_{3}=0.00195, c_{4}=-4.0319 \times 10^{-12} .
$$

Therefore, the approximate solution is given by

$$
x_{4}(t)=\sum_{n=0}^{4} c_{i} V_{i}^{*}(t)=0.70894 V_{0}^{*}(t)+0.251995 V_{1}^{*}(t)+0.03710 V_{2}^{*}(t)+0.00195 V_{3}^{*}(t)-4.0319 \times 10^{-12} V_{4}^{*}(t)
$$

The exact solution of this example is $x(t)=t^{3}$. The behavior of the approximate solution using the proposed method

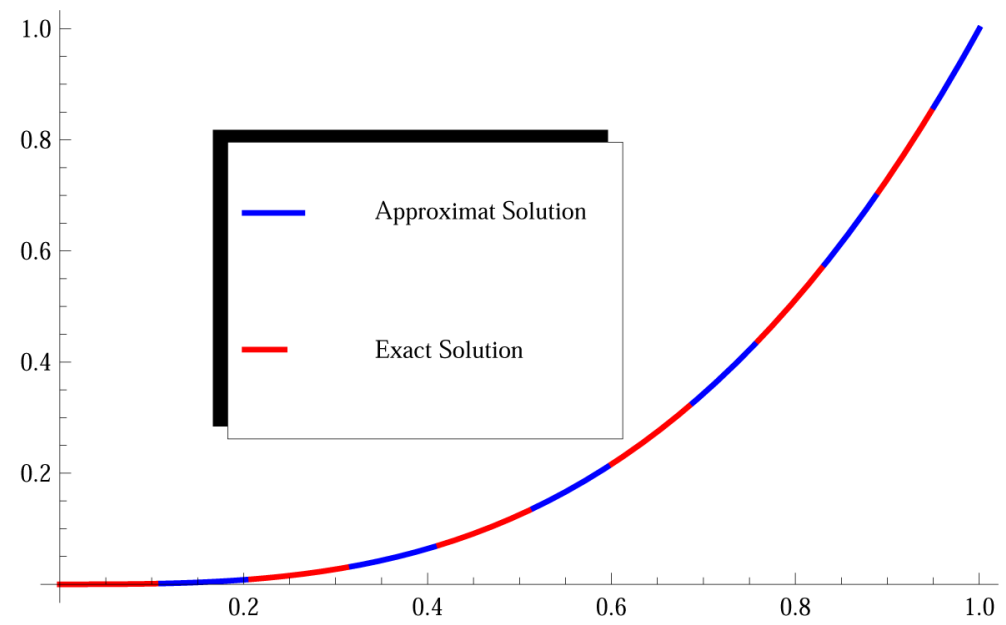

Fig. 1: The behavior of the exact solution and the approximate solution at $N=4$.

with $N=4$ and the exact solution in figure 1 . 
From this figure 1, its is clear that the proposed method can be considered as an efficient methods to solve the linear integral equation.

Example 2. Consider the following integral equation [17]

$$
x(t)=2 t e^{t}-e^{t}+1-\int_{0}^{t}(s+t) e^{x(s)} d s,
$$

where $f(t)=2 t e^{t}-e^{t}+1, k_{1}(t, s)=0, k_{2}(t, s)=s+t, \lambda_{1}=0, \lambda_{2}=-1 F(x(s))=0$ and $G(x(s))=e^{x(s)}$. We apply the suggested method with $N=4$ and approximate the solution $x(t)$ as follows

$$
x_{N}(t)=\sum_{i=0}^{4} c_{i} V_{i}^{*}(t)
$$

By the same procedure in the previous section and using Eq.(15), we have

$$
\sum_{i=0}^{4} c_{i} V_{i}^{*}\left(t_{j}\right)+\frac{h_{j}}{2}\left[\Omega\left(s_{0}\right)+\Omega\left(s_{m}\right)+2 \sum_{k=1}^{m-1} \Omega\left(s_{k}\right)\right]=f\left(t_{j}\right), j=0,1,2,3,4
$$

where the nodes $s_{i+1}=s_{i}+h, i=0,1, \cdots, m, s_{0}=0$ and $h_{j}=\frac{t_{j}}{m}$.

$$
\Omega(s)=\left(s+t_{j}\right) e^{\sum_{i=0}^{4} c_{j} V_{i}^{*}(s)}
$$

Eq.(23) presents non-linear system of $N+1$ algebraic equation in the coefficients $c_{i}$. By solving it by using the Newton iteration method with suitable initial solution. We obtain

$$
c_{0}=0.87499, c_{1}=0.125, c_{2}=-2.349 \times 10^{-8}, c_{3}=2.736 \times 10^{-9}, c_{4}=6.610 \times 10^{-10} .
$$

Therefore, the approximate solution as follows

$$
x_{4}(t)=\sum_{n=0}^{4} c_{i} V_{i}^{*}(t)=0.87499 V_{0}^{*}(t)+0.125 V_{1}^{*}(t)-2.349 \times 10^{-8} V_{2}^{*}(t)+2.736 \times 10^{-9} V_{3}^{*}(t)+6.610 \times 10^{-10} V_{4}^{*}(t)
$$

The exact solution of this example is $x(t)=t$. The behavior of the approximate solution using the proposed method with $N=4$ and the exact solution are present in in figure 2. From this figure 2, its is clear that the proposed method can be considered as an efficient methods to solve the non-linear integral equation.

Example 3. Consider the following integral equation [17]

$$
x(t)=t e+1-\int_{0}^{1}(s+t) e^{x(s)} d s
$$

The exact solution of this problem is $x(t)=t$.

We apply the suggested method with $N=4$ and approximate the solution $x(t)$ as in Eq.(24) and the same procedure in the previous section and using Eq.(15), we have

$$
\sum_{i=0}^{3} c_{i} V_{i}^{*}\left(t_{j}\right)+\frac{h}{2}\left[\Omega\left(s_{0}\right)+\Omega\left(s_{m}\right)+2 \sum_{k=1}^{m-1} \Omega\left(s_{k}\right)\right]=f\left(t_{j}\right), \quad j=0,1,2,3
$$




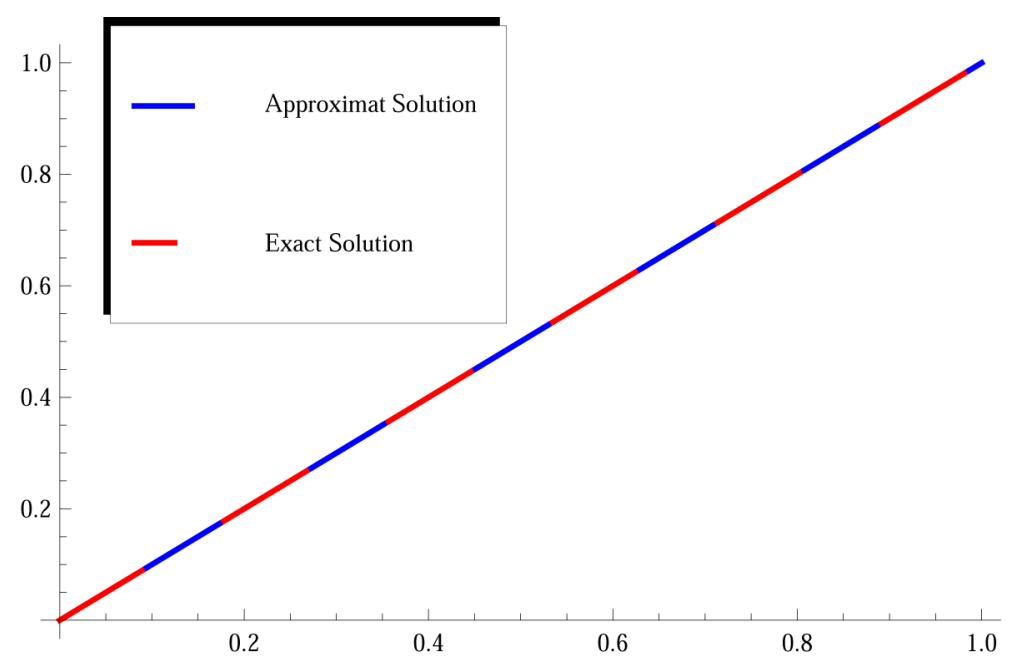

Fig. 2: The behavior of the exact solution and the approximate solution at $N=4$.

where the nodes $s_{i+1}=s_{i}+h, i=0,1, \cdots, m, s_{0}=0$ and $h=\frac{1}{m}$ and

$$
\Omega(s)=\left(s+t_{j}\right) e^{\sum_{i=0}^{3} c_{j} V_{i}^{*}(s)}
$$

Eq.(25) presents non-linear system of algebraic. By solving it by using the well known Newton iteration method with suitable initial solution. we obtain

$$
c_{0}=0.875, c_{1}=0.125, c_{2}=-2.4120 \times 10^{-16}, c_{3}=7.167 \times 10^{-17}, c_{4}=3.527 \times 10^{-17} .
$$

Therefore, the approximate solution of this example can be found using Eq.(24) as follows

$$
x_{4}(t)=\sum_{n=0}^{4} c_{i} V_{i}^{*}(t)=0.875 V_{0}^{*}(t)+0.125 V_{1}^{*}(t)-2.4120 \times 10^{-16} V_{2}^{*}(t)+7.167 \times 10^{-17} v_{3}^{*}(t)+3.527 \times 10^{-17} V_{4}^{*}(t)
$$

The exact solution of this example is $x(t)=t$. The behavior of the approximate solution using the proposed method with $N=4$ and the exact solution are present in in figure 3 . From this figure 3 , its is clear that the proposed method can be considered as an efficient methods to solve the non-linear integral equation.

Example 4. Consider the following integral equation [17]

$$
x(t)=\frac{t}{2}-\frac{t^{3}}{6}-\frac{1}{3}+\int_{0}^{1}(s+t) x(s) d s+\int_{0}^{t}(s-t) x(s) d s
$$

The exact solution of this problem is $x(t)=t$. We apply the suggested method with $N=4$ and approximate the solution $x(t)$ as follows

$$
x_{N}(t)=\sum_{i=0}^{4} c_{i} v_{i}^{*}(t)
$$




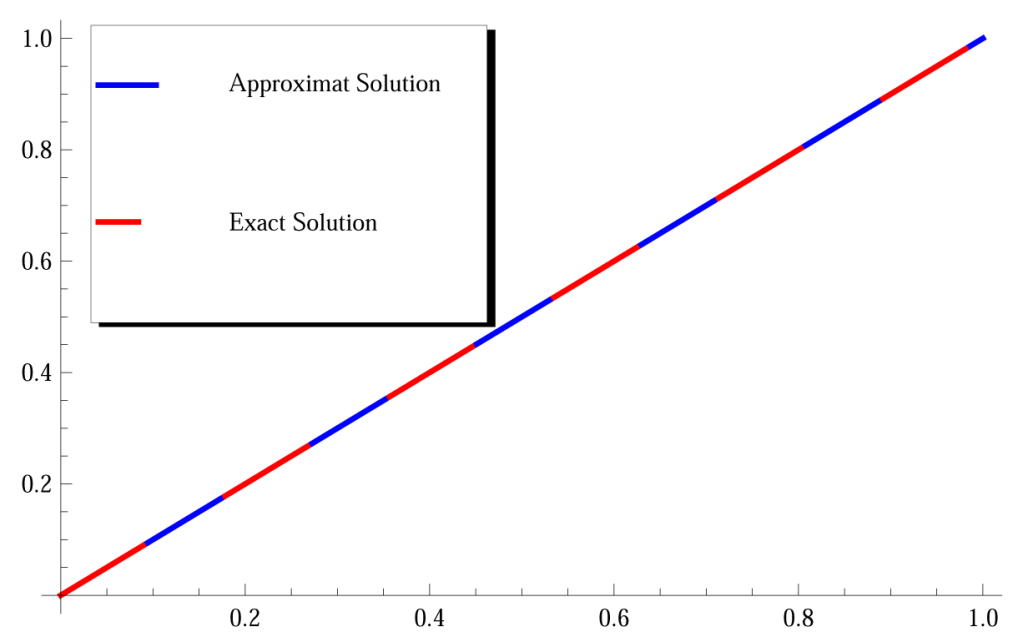

Fig. 3: The behavior of the exact solution and the approximate solution at $N=4$.

By the same procedure in the previous section and using Eq.(15), we have

$$
\sum_{i=0}^{4} c_{i} V_{i}^{*}\left(t_{j}\right)-\frac{h}{2}\left[\Omega_{1}\left(\bar{s}_{0}\right)+\Omega_{1}\left(\bar{s}_{m}\right)+2 \sum_{k=1}^{m-1} \Omega_{1}\left(\bar{s}_{k}\right)\right]-\frac{h_{j}}{2}\left[\Omega_{2}\left(\bar{s}_{0}\right)+\Omega_{2}\left(\bar{s}_{m}\right)+2 \sum_{k=1}^{m-1} \Omega_{2}\left(\bar{s}_{k}\right)\right]=f\left(t_{j}\right), \quad j=0,1,2,3,4
$$

where $\bar{s}_{t+1}=\bar{s}_{t}+h, s_{t+1}=s_{t}+h_{j}, i=0,1,2, \cdots, m, s_{0}=\bar{s}_{0}=0, h=\frac{1}{m}, h_{j}=\frac{t_{j}}{m}$ and $\Omega_{1}(s)=\left(s+t_{j}\right) \sum_{i=0}^{4} c_{i} V_{i}^{*}(s), \Omega_{2}(s)=$ $\left(t_{j}-s\right) \sum_{i=0}^{4} c_{i} v_{i}^{*}(s)$. Eq.(29) presents linear system $N+1$ algebraic equations. By solving it using Newton iteration method, we obtain

$$
c_{0}=0.875011, c_{1}=0.125, c_{2}=6.30883 \times 10^{-8}, c_{3}=8.3096 \times 10^{-11}, c_{4}=8.4650 \times 10^{-11} .
$$

Therefore, the approximate solution as follows

$x_{4}(t)=\sum_{n=0}^{4} c_{i} V_{i}^{*}(t)=0.875011 V_{0}^{*}(t)+0.125 V_{1}^{*}(t)+6.30883 \times 10^{-8} V_{2}^{*}(t)+8.3096 \times 10^{-11} V_{3}^{*}(t)+8.4650 \times 10^{-11} V_{4}^{*}(t)$

The behavior of the approximate solution using the proposed method with $N=4$ and the exact solution are present in in figure 4. From this figure 4, its is clear that the proposed method can be considered as an efficient methods to solve the linear Hammerstein integral equations.

Example 5. Consider the following integral equation [19]

$$
x(t)=e^{t+1}-\int_{0}^{1}\left(x^{3}(s)\right) e^{(t-2 s)} d s,
$$

where $f(t)=e^{t+1}$ and $k_{2}(t, s)=0, k_{1}(t, s)=e^{(t-2 s)}, \lambda_{1}=-1, \lambda_{2}=0 F(x(s))=x^{3}(s), G(x(s))=0$. It is and easy to verify that the exact solution of this problem is $x(t)=e^{t}$. We apply the suggested method with $N=4$ and approximate the solution $x(t)$ as follows

$$
x_{4}(t)=\sum_{i=0}^{4} c_{i} V_{i}^{*}(t)
$$




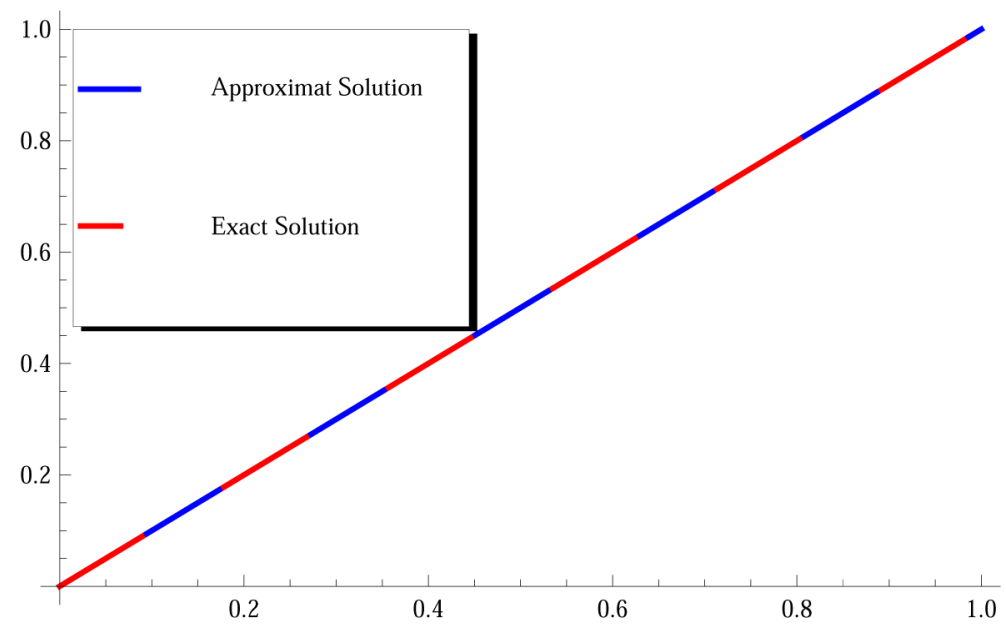

Fig. 4: The behavior of the exact solution and the approximate solution at $N=4$.

By the same procedure in the previous section and using Eq.(15), we have

$$
\sum_{i=0}^{4} c_{i} V_{i}^{*}\left(t_{j}\right)+\frac{h}{2}\left[\Omega\left(s_{0}\right)+\Omega\left(s_{m}\right)+2 \sum_{k=1}^{m-1} \Omega\left(s_{k}\right)\right]=f\left(t_{j}\right), \quad j=0,1,2,3,4
$$

where the nodes $s_{i+1}=s_{i}+h, i=0,1, \cdots, m, s_{0}=0$ and $h=\frac{1}{m}$.

$$
\Omega(s)=e^{\left(t_{j}-2 s\right)}\left(\sum_{i=0}^{4} c_{j} V_{i}^{*}(s)\right)^{3}
$$

Eq.(32) presents non-linear system of $N+1$ algebraic equation in the coefficients $c_{i}$. By solving it by using the Newton iteration method with suitable initial solution, we obtain

$$
c_{0}=2.41690, c_{1}=0.283323, c_{2}=0.0173170, c_{3}=0.0007112644, c_{4}=0.0000216190 .
$$

Therefore, the approximate solution as follows

$$
x_{4}(t)=\sum_{n=0}^{4} c_{i} V_{i}^{*}\left(t=2.41690 V_{0}^{*}(t)+0.283323 V_{1}^{*}(t)+0.0173170 V_{2}^{*}(t)+0.0007112644 V_{3}^{*}(t)+0.0000216190 V_{4}^{*}(t)\right.
$$

The behavior of the approximate solution using the proposed method with $N=4$ and the exact solution are present in in figure 5. From this figure 5, its is clear that the proposed method can be considered as an efficient method.

\section{Conclusion}

In this paper, we approximate method for the solution of linear and non-linear Fredholm and Volterra integral equations in the most general form has been proposed and investigated. The presented method which is based on the shifted Chebyshev polynomials of the third kind is proposed. A comparison of the exact solution reveals that the presented method is very effective and convenient. The numerical results show that the accuracy improves with increasing $\mathrm{N}$, hence for better results, using number $\mathrm{N}$ is recommended. Also, from the obtained approximate solution, we can conclude that 


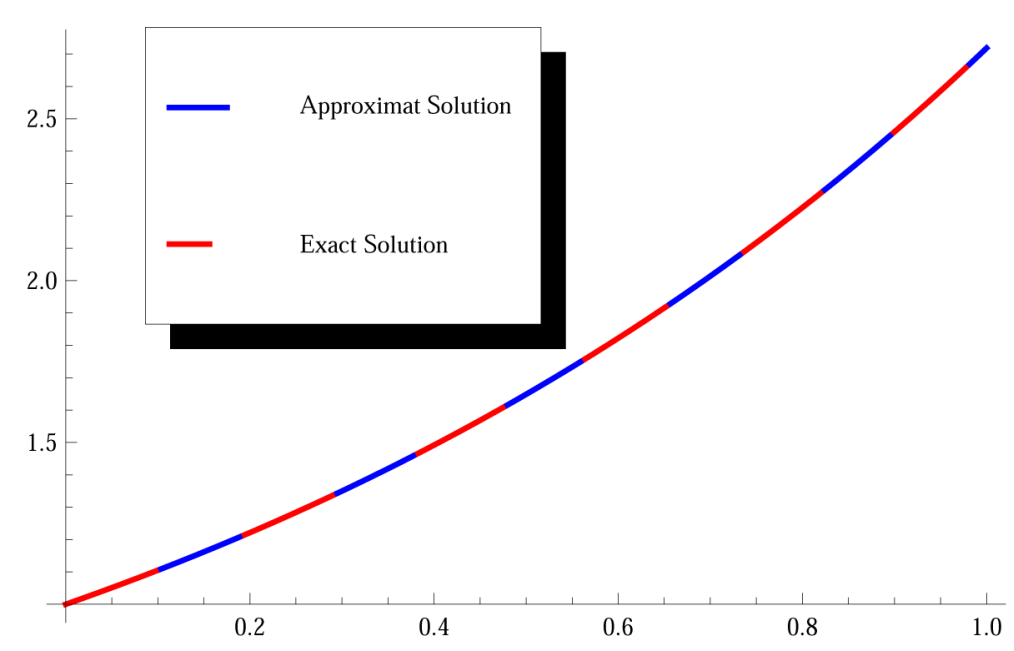

Fig. 5: The behavior of the exact solution and the approximate solution at $N=4$.

Table 1: Comparison between Exact solution and approximate solution and error for Example 5

\begin{tabular}{|c|c|c|c|}
\hline $\mathrm{X}$ & Exact solution & Approximate soiution & Error \\
\hline 0 & 1 & 1 & 0 \\
0.1 & 1.10517 & 1.10517 & $1.75803 \times 10^{-6}$ \\
0.2 & 1.2142 & 1.2142 & $3.32845 \times 10^{-7}$ \\
0.3 & 1.34986 & 1.34986 & $1.07348 \times 10^{-6}$ \\
0.4 & 1.49182 & 1.49182 & $4.06538 \times 10^{-7}$ \\
0.5 & 1.69872 & 1.69872 & $6.91612 \times 10^{-8}$ \\
0.6 & 1.82212 & 1.82212 & $4.96547 \times 10^{-7}$ \\
0.7 & 2.01375 & 2.01375 & $1.29584 \times 10^{-6}$ \\
0.8 & 2.22554 & 2.22554 & $6.06484 \times 10^{-7}$ \\
0.9 & 2.45966 & 2.45966 & $1.63799 \times 10^{-6}$ \\
1 & 2.71828 & 2.71828 & $7.40761 \times 10^{-7}$ \\
\hline
\end{tabular}

the proposed method gives the solution in an excellent agreement with the exact solution. All computations are done using Mathematica 7 programs.

\section{Competing interests}

The authors declare that they have no competing interests.

\section{Authors' contributions}

All authors have contributed to all parts of the article. All authors read and approved the final manuscript.

\section{References}

[1] R. P. Agarwal, Boundary value problems for higher order integro- differential equations, Nonlinear Anal. Theory Methods Appl., 9 (1983), 259-270.

[2] L. C. Andrews, Special Functions For Engineers and Applied Mathematicians, Macmillan publishing company, New York, (1985). 
[3] E. Babolian, F. Fattahzadeh, E. Golpar Raboky, A Chebyshev approximation for solving nonlinear integral equations of Hammerstein type, Applied Mathematics and Computation, 189 (2007), 641-46.

[4] A. K. Borzabadi, A. V. Kamyad and H. H. Mehne, A different approach for solving the nonlinear Fredholm integral equations of the second kind, Applied Mathematics and Computation, 173 (2006), 724-735.

[5] L. M. Delves and J. L. Mohamad, Computational Methods for Integral Equations, Cambridge University press, (1985).

[6] M. M. Khader and A. S. Hendy, The approximate and exact solutions of the fractional-order delay differential equations using Legendre pseudospectral method, International Journal of Pure and Applied Mathematics, 74(3) (2012), 287-297.

[7] M. M. Khader, N. H. Sweilam and A.M.S. Mahdy, An efficient numerical method for solving the fractional diffusion equation, Journal of Applied Mathematics and Bioinformatics, 1 (2011), 1-12.

[8] M. M. Khader, Introducing an efficient modification of the homotopy perturbation method by using Chebyshev polynomials, Arab Journal of Mathematical Sciences, 18 (2012), 61-71.

[9] M. M. Khader, Numerical solution of nonlinear multi-order fractional dif- ferential equations by implementation of the operational matrix of frac- tional derivative, Studies in Nonlinear Sciences, 2(1) (2011), 5-12.

[10] S. T. Mohamed and M. M. Khader, Numerical solutions to the second order Fredholm integro-differential equations using the spline functions expansion, Global Journal of Pure and Applied Mathematics, 34 (2011), 21-29.

[11] N. Kurt and M. Sezer, Polynomial solution of high-order linear Fredholm integro-differential equations with constant coeffcients, Journal of the Franklin Institute, 345 (2008), 839-850.

[12] M. Shahrezaee, Solving an integro-differential equation by Legendre polynomials and Block-pulse functions, Dynamical Systems and Applications, (2004), 642-647.

[13] N. H. Sweilam, Fourth order integro-differential equations using variational iteration method, Comput. Maths. Appl., 54 (2007), 1086-1091.

[14] N. H. Sweilam, M. M. Khader and R.F. Al-Bar, Homotopy perturbation method for linear and nonlinear system of fractional integro-differential equations, International Journal of Computational Mathematics and Numerical Simulation, 1 (2008), $73-87$.

[15] N. H. Sweilam and M. M. Khader, A Chebyshev pseudo-spectral method for solving fractional order integro-differential equations, ANZIAM, 51 (2010), 464-475.

[16] N. H. Sweilam and M. M. Khader, Semi exact solutions for the bi-harmonic equation using homotopy analysis method, World Applied Sciences Journal, 13 (2011), 1-7.

[17] N. H. Sweilam, M. M. Khader, and W. Y. Kota, On the Numerical Solution of Hammerstein Integral Equations using Legendre Approximation International Journal of Applied Mathematical, Research.1(1) (2012), 65-76

[18] S. Youse and M. Razzaghi, Legendre wavelet method for the nonlinear Volterra-Fredholm integral equations, Math. Comp. Simul., 70 (2005), 1-8.

[19] Z. Esmailzadeh and A. Jafarian, On the Numerical Solution of Urysohn Integral Equations using Legendre Approximation, Journal of Mathematical Modeling, 1(2013),76-84.

[20] N. H. Sweilam, A. M. Nagy and A. A. El-Sayed, On the numerical solution of space fractional order diffusion equation via shifted Chebyshev polynomials of the third kind, Journal of King Saud University Science, 28, (2016), 41-47. 